\title{
Risk Factors for Early Death After Rituximab- Based Immunochemotherapy in Older Patients With Diffuse Large B-Cell Lymphoma
}

\author{
Adam J. Olszewski, MD ${ }^{\mathrm{a}, \mathrm{b}}$; Kalyan C. Mantripragada, MD, MPH ${ }^{\mathrm{a}, \mathrm{b}}$; and Jorge J. Castillo, MDc
}

\begin{abstract}
Background: Older patients with diffuse large B-cell lymphoma (DLBCL) are at risk of severe chemotherapy-related morbidity and mortality. Our objective was to quantify the risk and identify factors associated with death during the first cycle of immunochemotherapy in this population. Patients and Methods: Using Medicare claims linked to the population-based SEER registry (SEER-Medicare), we studied patients with DLBCL aged 65 years and older who received immunochemotherapy containing rituximab, cyclophosphamide, and vincristine, in combination with doxorubicin, mitoxantrone, or etoposide in 2003-2012. Risk factors for death and hospitalization within the first 30 days of treatment were studied in multivariable logistic regression models. Results: We identified 5,530 patients with a median age of 76 years, of whom $94 \%$ received doxorubicin-containing immunochemotherapy. Granulocyte colony-stimulating factor (G-CSF) was administered to $66 \%$ of patients during the first treatment cycle. Cumulative incidence of death at day 30 was $2.2 \%$. The risk was significantly higher in patients aged 75 years and older and those who had B symptoms, chronic kidney disease, poor functional status, use of walking aids or wheelchairs, and prior hospitalization or upper endoscopy. The group with 0 to 1 risk factors ( $56 \%$ of patients) had a very low ( $0.6 \%$ ) risk of early death, whereas the group with 4 or more risk factors ( $6 \%$ of patients) had a risk of $8.3 \%$. Receipt of G-CSF was associated with a lower probability of early death in the high-risk group. The incidence of hospitalization within the first 30 days was $23.5 \%$, peaking at day 8 of the cycle. Conclusions: Among older patients with DLBCL who receive contemporary immunochemotherapy, 1 in 45 die during the first month of treatment, and 1 in 4 are hospitalized. Factors identifiable from administrative/electronic records can stratify this risk and could be incorporated into decision support tools. Prophylactic G-CSF is not administered to more than one-third of patients, indicating an opportunity for improved preventive interventions.
\end{abstract}

J Natl Compr Canc Netw 2016;14(9):1121-1129

\section{Background}

Diffuse large B-cell lymphoma (DLBCL) is a curable malignancy for approximately $60 \%$ of patients aged 65 years or older, if treated with contemporary immunochemotherapy. ${ }^{1}$ However, the risk of severe toxicities and death after chemotherapy initiation significantly increases with age. ${ }^{2,3}$ Even among preselected clinical trial participants, approximately $5 \%$ die of treatment-

From the a Department of Medicine, Alpert Medical School of Brown University, and 'Division of Hematology-Oncology, Rhode Island Hospital, Providence, Rhode Island; and 'Division of Hematologic Malignancies, Dana-Farber Cancer Institute, Harvard Medical School, Boston, Massachusetts.

Submitted April 21, 2016; accepted for publication June 1, 2016. Results of this study were presented in part at the 57th American Society of Hematology Annual Meeting \& Exposition; December 4-8, 2015; Orlando, FL.

Dr. Olszewski has disclosed that he receives consulting fees from BristolMyers Squibb and institutional research support from Genentech and TG Therapeutics, and is supported by the ASH Research scholar award. Dr. Mantripragada has disclosed that he has no financial interests, related complications. ${ }^{4-7}$ In order to lower this risk, oncologists rely on clinical intuition or institutional practice patterns, balancing the intensity of curative therapy with potential toxicity. A quarter of Medicare beneficiaries diagnosed with DLBCL may not receive chemotherapy at all. ${ }^{8}$ Identifying the risk of severe complications is challenging given the paucity of data and heterogeneity in physiologic reserve among patients of

arrangements, affiliations, or commercial interests with the manufacturers of any products discussed in this article or their competitors. Dr. Castillo has disclosed that he receives consulting fees from Otsuka and research funding from Millennium, Pharmacyclics, and Gilead Sciences. This research was funded by Alpert Medical School of Brown University. Author contributions: Study design: Olszewski and Mantripragada. Analysis: Olszewski and Mantripragada. Data interpretation: Olszewski, Mantripragada, and Castillo. Manuscript writing and approval: Olszewski, Mantripragada, and Castillo.

Correspondence: Adam J. Olszewski, MD, Alpert Medical School of Brown University, Comprehensive Cancer Center at Rhode Island Hospital, 593 Eddy Street, George 301, Providence, RI 02903.

E-mail: adam_olszewski@brown.edu 
Olszewski et al

the same age..$^{9,10}$ Validated tools such as the comprehensive geriatric assessment (CGA) are too complex for many practice settings, and therefore discerning risk factors from readily available clinical or administrative data offers a useful alternative for designing preventive interventions. ${ }^{11,12}$

Most prior studies concentrated on adverse events during the entire course of chemotherapy, or on patients' ability to complete the planned number of cycles. ${ }^{8,13,14}$ However, the initial treatment cycle is of critical importance because patients' functional status, nutrition, and organ function are impaired by active lymphoma. Our objective was to evaluate risk factors for death and hospitalization during the first month of rituximab-based immunochemotherapy using population-based data from Medicare beneficiaries.

\section{Patients and Methods}

\section{Data Source and Cohort Selection}

This study used deidentified Medicare claims combined with SEER program data (SEER-Medicare), and was approved by the local Institutional Review Board. The dataset links cancer registry records from 18 geographic areas encompassing 28\% of the US population, with claims for health services rendered to Medicare beneficiaries. It has been extensively used for health outcomes research on older Americans with DLBCL. ${ }^{8,15-18}$ We selected patients diagnosed with DLBCL at age 65 years or older between 2003 and 2011 who initiated outpatient immunochemotherapy containing, at minimum, rituximab, cyclophosphamide, and vincristine in combination with doxorubicin, mitoxantrone, or etoposide (Figure 1). We excluded cases with incomplete Medicare records-primarily enrollees in managed care plans, whose billing claims are not processed by Medicare administration. We also excluded subjects with primary central nervous system lymphoma, and those who did not receive treatment within 3 months from diagnosis. HIV status was evident from claims in only fewer than 11 cases.

\section{Variables and End Points}

We classified race/ethnicity as white non-Hispanic, white Hispanic, black, or Asian/other. Dual coverage by Medicare and Medicaid, available specifically to low-income beneficiaries, served as a proxy for socioeconomic deprivation. For adjustment of

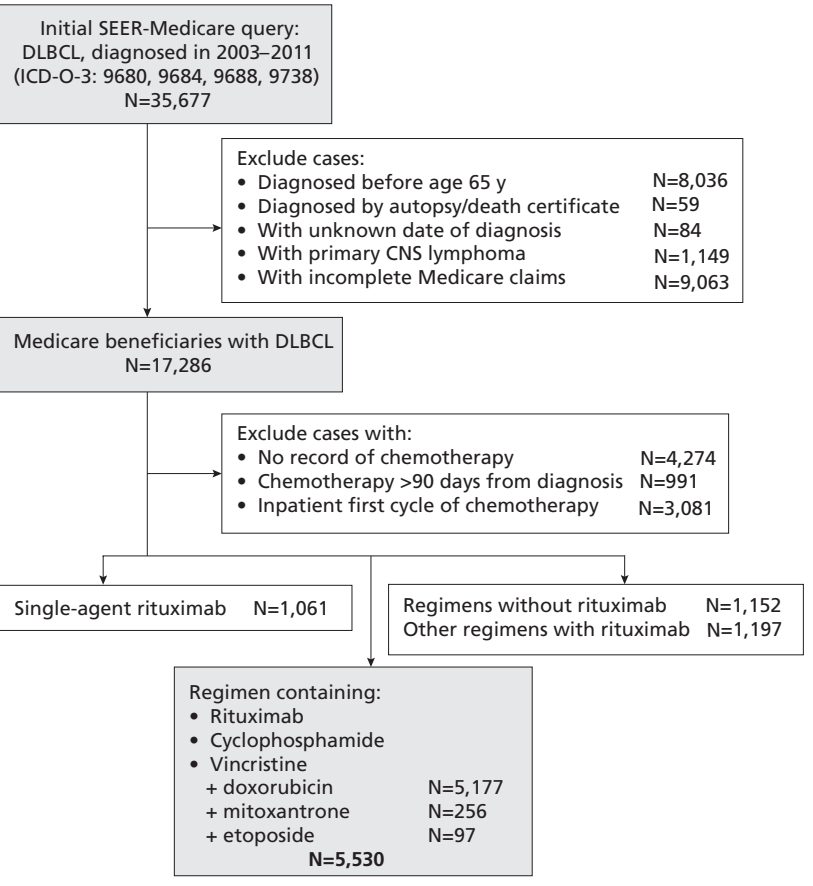

Figure 1. Selection of the analytic cohort from the SEER-Medicare data.

Abbreviations: CNS, central nervous system; DLBCL, diffuse large B-cell lymphoma.

baseline health, we used 2 claims-based constructs. The NCI modification of the Charlson comorbidity index, based on diagnostic codes within 1 year before diagnosis, counts medical conditions associated with baseline mortality and receipt of chemotherapy. ${ }^{8,15,19,20}$ Davidoff's disability indicator, derived from use of health services before diagnosis, is a validated measure of self-reported poor performance status (PS; corresponding to ECOG 3-4 category). ${ }^{21,22} \mathrm{We}$ also studied selected comorbidities and health services using claims from 1 year preceding the DLBCL diagnosis. In particular, chronic kidney disease was defined as occurrence of any of the following codes from the ICD-9: 582.*, 583.*, 585.*, 586, or 588** Health services were identified using Berenson-Eggers Type of Service algorithms for Medicare claims analysis. ${ }^{23}$

Chemotherapy regimen was ascertained from codes for specific drugs within 7 days from the first administration. Doses of agents were not available. We classified the regimens as R-CHOP-like (containing rituximab, doxorubicin, cyclophosphamide, and vincristine), or with mitoxantrone or etoposide substituting for doxorubicin. The use of steroids was assumed, because oral prescriptions were not re- 
Early Death in Older Patients With DLBCL

corded. We also identified prophylactic granulocyte colony-stimulating factor (G-CSF) administration during the first cycle. ${ }^{24}$

The primary outcome of analysis was death within 30 days from chemotherapy initiation. The secondary outcome was hospitalization. We also identified admissions to critical care units or nursing homes, and cardiovascular events ascertained from inpatient Medicare files within the same time frame.

\section{Statistical Analysis}

Univariate associations were analyzed by chi-square test. We then built multivariable logistic models, always including age, sex, race, DLBCL stage, B symptoms, comorbidity score, PS indicator, Medicaid coverage, and receipt of anthracycline as covariates, regardless of statistical significance. Other predictors showing strong association $(P<.002$ - to correct for multiple testing) were added stepwise and retained in the final model if they remained significant $(P<.05)$. Missing data on stage and $B$ symptoms were accounted for by multiple imputation using chained equations that included all variables from outcome models, generating 30 imputed data sets. ${ }^{25}$ Time-dependent hazards were calculated in flexible parametric survival models. ${ }^{26}$ Analyses were conducted using SAS 9.4 (SAS Institute Inc., Cary, NC) or Stata/MP 14.1 (StataCorp LP, College Station, TX) and report estimates with 95\% CIs.

\section{Results}

\section{Patient Characteristics}

Among the 5,530 eligible patients (with a median age of 76 years) treated with outpatient rituximabbased immunochemotherapy in 2003-2011 (Table 1), $94 \%$ received anthracycline, and $66 \%$ received G-CSF during the first cycle. With a median followup of 5.7 years, median overall survival (OS) was 7.0 years (95\% CI, 6.8-7.4), and the estimated 5-year survival rate was $60 \%(\mathrm{CI}, 58 \%-61 \%)$. This was better for patients who received doxorubicin $(61 \%$; CI, $59 \%-62 \%)$ compared with those who did not (43\%; CI, 38\%-49\%). Excluded patients treated with less intense regimens also had worse survival rates $(38 \%$; CI, 36\%-39\%). The hazard of death peaked at 30 days from the first chemotherapy (Figure 1A) and plateaued after approximately 30 months.

\begin{tabular}{|c|c|c|}
\hline Variable & $\mathbf{N}$ & $\%$ \\
\hline Number of cases & 5,530 & \\
\hline \multicolumn{3}{|l|}{ Age } \\
\hline $65-69$ y & 1,057 & 19.1 \\
\hline $70-74$ y & 1,494 & 27.0 \\
\hline $75-79$ y & 1,466 & 26.5 \\
\hline$\geq 80 y$ & 1,513 & 27.4 \\
\hline \multicolumn{3}{|l|}{ Sex } \\
\hline Women & 2,721 & 49.2 \\
\hline Men & 2,809 & 50.8 \\
\hline \multicolumn{3}{|l|}{ Race/ethnicity } \\
\hline White non-Hispanic & 4,837 & 87.5 \\
\hline White Hispanic & 284 & 5.1 \\
\hline Black & 157 & 2.8 \\
\hline Asian/other & 252 & 4.6 \\
\hline \multicolumn{3}{|l|}{ Medicaid coverage } \\
\hline Yes & 503 & 9.1 \\
\hline No & 5,027 & 90.9 \\
\hline \multicolumn{3}{|l|}{ Charlson comorbidity index } \\
\hline 0 & 3,029 & 54.8 \\
\hline 1 & 1,430 & 25.9 \\
\hline 2 & 614 & 11.1 \\
\hline 3 & 274 & 5.0 \\
\hline$\geq 4$ & 183 & 3.3 \\
\hline \multicolumn{3}{|l|}{ Performance status } \\
\hline Not poor & 5,261 & 95.1 \\
\hline Poor & 269 & 4.9 \\
\hline \multicolumn{3}{|l|}{ Ann Arbor stage } \\
\hline 1 & 1,702 & 30.8 \\
\hline 2 & 1,166 & 21.1 \\
\hline 3 & 960 & 17.4 \\
\hline 4 & 1,399 & 25.3 \\
\hline Unrecorded & 303 & 5.5 \\
\hline \multicolumn{3}{|l|}{ B symptoms } \\
\hline Absent & 3,015 & 54.5 \\
\hline Present & 993 & 18.0 \\
\hline Unrecorded & 1,522 & 27.5 \\
\hline \multicolumn{3}{|l|}{ Primary site } \\
\hline Nodal & 3,636 & 65.8 \\
\hline Extranodal & 1,894 & 34.2 \\
\hline Prophylactic G-CSF given & 3,630 & 65.6 \\
\hline Radiation before chemotherapy & 152 & 2.7 \\
\hline
\end{tabular}

Abbreviation: G-CSF, granulocyte colony-stimulating factor. 


\section{Risk of Early Death}

The cumulative incidence of death was $2.2 \%(\mathrm{CI}$, $1.8 \%-2.6 \%$ ) at 30 days, and $10.9 \%$ (CI, $10.1 \%-$ $11.8 \%)$ at 180 days from chemotherapy initiation (Figure 1B). The most common causes of death (from death certificates) within the first 30 days were lymphoma (72\%), heart disease (9\%), septicemia (3\%), and cerebrovascular events (3\%). In univariate analysis, risk significantly increased with age, presence of B symptoms, nonreceipt of anthracycline, number of comorbidities, worsening PS, and several claim-derived predictors (Table 2 and supplemental eTable 1, available with this article at JNCCN.org). We constructed a simplified prediction score based on 7 factors significant in the multivariable model: age of 75 years or older, B symptoms, poor PS, chronic kidney disease, use of mobility aids (cane, walker, wheelchair), and hospitalization or upper endoscopy within 1 year before diagnosis. This score classified $56 \%$ of patients as low risk ( $0-1$ factors) with $0.6 \%$ (CI, $0.3 \%-0.9 \%$ ) chance of early death, and an additional $38 \%$ of patients as intermediate risk (2-3 factors, $3.2 \%$ risk of early death; CI, $2.5 \%-4.0 \%)$. It also discerned a small $(6 \%)$ high-risk group ( $\geq 4$ factors) with a probability of early death of $8.3 \%(\mathrm{CI}$, 5.3\%-11.0\%; Figure 2C, D). This discrimination was similar for patients aged 80 years or older (risk of $0.9 \%, 4.4 \%$, and $7.8 \%$ for low-, intermediate-, and high-risk groups, respectively) and for younger patients $(0.6 \%, 2.6 \%$, and $8.8 \%$, respectively). Among patients aged 80 years or older, $40 \%$ were in the lowrisk and $10 \%$ were in the high-risk category.

The score also correlated with the risk of death at 180 days $(6 \%, 15 \%$, and $27 \%$ for low-, intermediate-, and high-risk groups, respectively) and with subsequent OS (supplemental eFigure 1). Furthermore, although not calibrated for this group, the score was associated with the risk of early death among patients treated with low-intensity regimens (supplemental eTable 2).

In order to eliminate guarantee-time bias, we studied the association between receipt of G-CSF and early death in a subgroup of patients $(\mathrm{N}=5,478)$ who remained alive and as outpatients within 2 days after chemotherapy (Table 3). ${ }^{27}$ Prophylactic GCSF was not administered to $36 \%, 32 \%$, and $33 \%$ of patients in the low-, intermediate-, and high-risk groups, respectively. The risk of early death was similar with or without G-CSF in low- and intermediate- risk groups, but was 3 times higher among high-risk patients who did not receive G-CSF (15\% vs 5\%; $P=.001)$.

\section{Risk of Hospitalization}

Cumulative incidence of hospitalization within 30 days of chemotherapy was $23.5 \%$ (CI, 22.4\%$24.6 \%$ ), and the risk peaked on day 8 (Figure 3A). In a multivariable model, risk was associated with increasing age, male sex, B symptoms, comorbidity index, and prior hospitalization or upper endoscopy, but not with any specific comorbidity or anthracycline use (supplemental eTable 3). Within the previously defined risk categories for early death, the probability of hospitalization varied from $18 \%$ to $40 \%$ (Figure 3B). Furthermore, $8 \%$ of patients were admitted to a critical care unit and $2 \%$ to a nursing home; $18 \%$ had a cardiovascular event, including $17 \%$ of those who received doxorubicin and $25 \%$ of those who did not. Among patients surviving more than 30 days, hospitalization during the first cycle was associated with a higher risk of not receiving further chemotherapy ( $9 \%$ vs $<1 \% ; P<.0001)$ and a higher risk of second-cycle delay beyond 22 days (50\% vs $32 \% ; P<.0001$ ).

\section{Discussion}

In this population-based study, we evaluated the risk of death or hospitalization during the first month of R-CHOP-like immunochemotherapy among Medicare beneficiaries with DLBCL. We found that 1 in 45 of those patients died and 1 in 4 was hospitalized. We distinguished groups with risk of death ranging from $0.6 \%$ to $8.3 \%$, which can help in the design of decision support tools and preventive interventions, and allow for evidence-driven counseling at the critical moment of therapy initiation. Supplemental eFigure 2 shows a simple applicable patient counseling tool. Considering the cumulative risk of death of $10.9 \%$ at 6 months, we can estimate that approximately $20 \%$ of treatment-related mortality occurs during the first month of chemotherapy.

Although advanced age at diagnosis is an important negative prognostic factor in DLBCL, ${ }^{28,29}$ in randomized trials of R-CHOP immunochemotherapy focused on older patients, most participants achieved long-term survival. In the landmark Groupe d'Etude des Lymphomes de l'Adulte study, 
Early Death in Older Patients With DLBCL

\begin{tabular}{|c|c|c|c|c|c|}
\hline \multirow[b]{2}{*}{ Variable } & \multicolumn{2}{|c|}{ Univariate $\left(\chi^{2}\right)$} & \multicolumn{3}{|c|}{ Multivariate Logistic Model } \\
\hline & $\begin{array}{c}\text { Early Death } \\
(\%)\end{array}$ & $P$ Value & Odds Ratio & $95 \% \mathrm{Cl}$ & $P$ Value \\
\hline \multicolumn{6}{|l|}{ Age } \\
\hline $65-69$ y & 1.0 & .0001 & Reference & & .0044 \\
\hline $70-74$ y & 1.5 & & 1.42 & $(0.68-2.96)$ & \\
\hline $75-79$ y & 2.2 & & 1.89 & $(0.94-3.81)$ & \\
\hline$\geq 80 y$ & 3.4 & & 2.86 & $(1.46-5.60)$ & \\
\hline \multicolumn{6}{|l|}{ Sex } \\
\hline Men & 1.9 & .44 & Reference & & .38 \\
\hline Women & 2.2 & & 0.84 & $(0.57-1.24)$ & \\
\hline \multicolumn{6}{|l|}{ Race } \\
\hline White non-Hispanic & 2.2 & .42 & Reference & & .22 \\
\hline White Hispanic & 1.1 & & 0.33 & $(0.10-1.13)$ & \\
\hline Black & 1.9 & & 0.59 & $(0.17-1.99)$ & \\
\hline Asian/other & 1.2 & & 0.54 & $(0.16-1.77)$ & \\
\hline \multicolumn{6}{|l|}{ Medicaid coverage } \\
\hline Absent & 2.1 & .64 & Reference & & .74 \\
\hline Present & 2.4 & & 0.89 & $(0.44-1.80)$ & \\
\hline \multicolumn{6}{|l|}{ Ann Arbor stage } \\
\hline 1 & 1.4 & .15 & Reference & & .44 \\
\hline 2 & 2.1 & & 1.33 & $(0.74-2.39)$ & \\
\hline 3 & 2.5 & & 1.50 & $(0.83-2.71)$ & \\
\hline 4 & 2.6 & & 1.53 & $(0.89-2.63)$ & \\
\hline \multicolumn{6}{|l|}{ B symptoms } \\
\hline Absent & 1.6 & .0008 & Reference & & .008 \\
\hline Present & 3.6 & & 1.93 & $(1.19-3.13)$ & \\
\hline \multicolumn{6}{|l|}{ Anthracycline } \\
\hline Given & 2.0 & .0036 & Reference & & .11 \\
\hline Not given & 4.2 & & 1.63 & $(0.90-2.94)$ & \\
\hline \multicolumn{6}{|l|}{ Comorbidity index ${ }^{a}$} \\
\hline 0 & 1.6 & $<.0001$ & Reference & & .38 \\
\hline 1 & 2.3 & & 1.12 & $(0.70-1.78)$ & \\
\hline 2 & 2.1 & & 0.60 & $(0.30-1.21)$ & \\
\hline 3 & 3.3 & & 0.52 & $(0.21-1.28)$ & \\
\hline$\geq 4$ & 7.1 & & 0.75 & $(0.29-1.89)$ & \\
\hline \multicolumn{6}{|l|}{ Performance status ${ }^{a}$} \\
\hline Not poor & 1.8 & $<.0001$ & Reference & & .014 \\
\hline Poor & 7.1 & & 2.14 & $(1.16-3.93)$ & \\
\hline \multicolumn{6}{|l|}{ Chronic kidney disease ${ }^{a}$} \\
\hline No & 1.8 & $<.0001$ & Reference & & $<.0001$ \\
\hline Yes & 7.6 & & 5.38 & $(2.48-11.65)$ & \\
\hline \multicolumn{6}{|c|}{ Use of walking aids/wheelchair ${ }^{a}$} \\
\hline No & 1.8 & $<.0001$ & Reference & & .0034 \\
\hline Yes & 6.2 & & 2.19 & $(1.30-3.69)$ & \\
\hline \multicolumn{6}{|l|}{ Hospitalizationa } \\
\hline No & 1.2 & $<.0001$ & Reference & & .0011 \\
\hline Yes & 3.8 & & 2.02 & $(1.33-3.08)$ & \\
\hline \multicolumn{6}{|l|}{ Upper endoscopya } \\
\hline No & 1.6 & $<.0001$ & Reference & & .0008 \\
\hline Yes & 3.8 & & 1.97 & $(1.32-2.93)$ & \\
\hline
\end{tabular}

aBased on Medicare claims within 1 year before the diagnosis of lymphoma. 


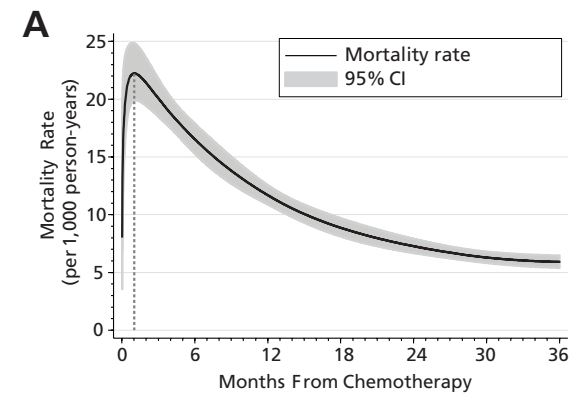

C

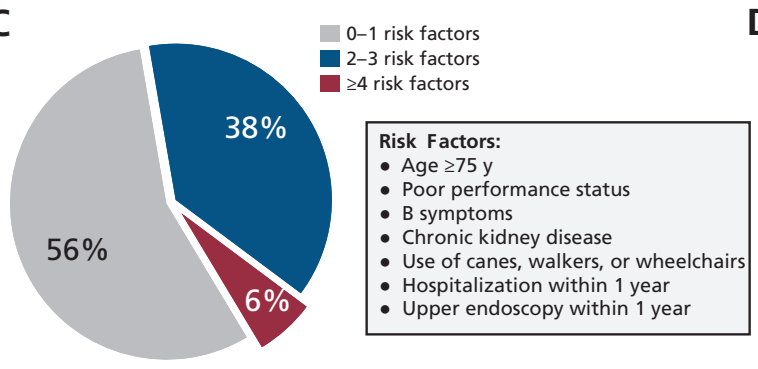

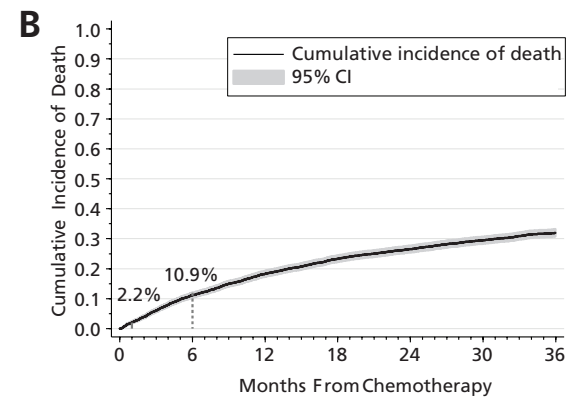

D

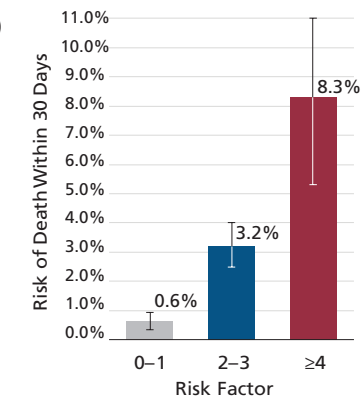

Figure 2. Analysis of early mortality in older patients with diffuse large B-cell lymphoma treated with rituximab-based immunochemotherapy. (A) Hazard of mortality as a function of time, indicating a peak at 30 days from the first treatment. (B) Cumulative incidence of death within 3 years from the first treatment. (C) Proportions of patients classified as low, intermediate, or high risk for early death, according to the number of risk factors. (D) Risk of death within 30 days from first treatment, stratified by number of risk factors; bars indicate $95 \% \mathrm{Cls}$.

the 5-year event-free survival rate after R-CHOP was $47 \%$ and the OS rate was $58 \% .{ }^{30}$ Furthermore, the 3 -year OS rate was $67 \%$ in the US Intergroup trial and $78 \%$ in the German RICOVER-60 trial (after 6 cycles of R-CHOP). ${ }^{6,7}$ These results come at a price of significant toxicities. In RICOVER-60, grade $3 / 4$ toxicities included infection $(28 \%)$, red blood cell transfusions (47\%), and arrhythmias (4\%). Similarly, Delarue et $\mathrm{al}^{4}$ found an $18 \%$ rate of febrile neutropenia and a $4 \%$ rate of cardiovascular severe adverse effects, with $13 \%$ of all fatalities ascribed to complications of R-CHOP. The balance between treatment intensity and tolerability thus becomes increasingly difficult to achieve in the geriatric population as age advances, functional reserve declines, and comorbidities accumulate. ${ }^{2,31}$ Elderly patients constitute a heterogeneous group, and age alone poorly correlates with treatment tolerance..$^{10,32}$ The cutoff of 75 years better reflects the prevalence of geriatric syndromes and functional dependence, but trials enroll few subjects beyond that age..$^{32,33}$ In our data, age of 75 years or older strongly correlated with early death or hospitalization. Surveys demonstrate that physicians variably offer or withhold curative chemotherapy from patients with DLBCL older than 80 years, which underscores the need for datadriven risk stratification. ${ }^{8,18,34,35}$ Our study fills in the gap in knowledge about mortality in the immediate period after R-CHOP initiation.

The CGA, a multidimensional assessment of medical, functional, nutritional, cognitive, and psychosocial domains, can help guide therapy in geriatric oncology, including predicting risk of early death. ${ }^{10,36,37}$ One prospective study evaluated CGAmodulated chemotherapy in 100 elderly patients with DLBCL, stratifying participants into fit, unfit, or frail groups, and modifying R-CHOP accordingly. ${ }^{12}$ The authors noted relatively low toxicity with a preserved 5-year OS rate of 60\%. However, application of the CGA in a wider clinical practice is hampered by its resource- and time-intensive nature. Two simpler tools combine elements of the CGA with standard oncologic workup. The Cancer and Aging Research Group (CARG) tool predicts a risk of grade 3 through 5 chemotherapy toxicity ranging from $25 \%$ to $89 \% .{ }^{38}$ This tool was derived largely from patients receiving palliative chemotherapy for solid tumors, thus its calibration for outcomes of R-CHOP immunochemotherapy is uncertain. The Chemotherapy Risk Assessment Scale for High-Age Patients (CRASH), based on clinical and laboratory parameters (including Mini-Mental and Mini-Nutritional assessments), can predict risk ranging from $7 \%$ to $100 \%$ for hematologic and $33 \%$ to $93 \%$ for nonhe- 
Early Death in Older Patients With DLBCL

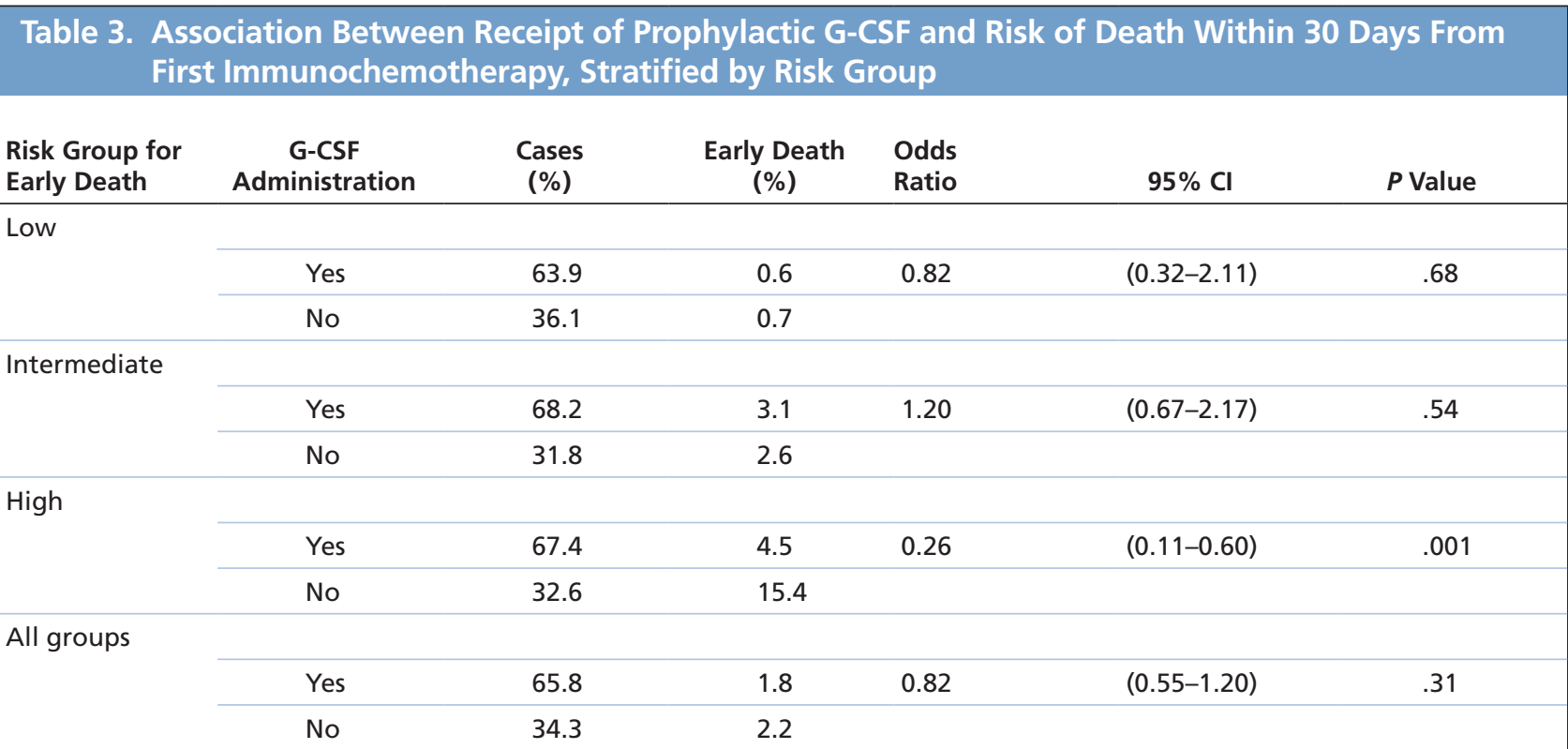

Abbreviations: DLBCL, diffuse large B-cell lymphoma; G-CSF, granulocyte colony-stimulating factor.

matologic toxicities. ${ }^{14}$ Both tools require calculation of subscores from dedicated clinical assessments, and therefore their wider applicability remains to be demonstrated. Our approach differs in that we focused on the immediate fatal toxicity during the first cycle of immunochemotherapy, and relied on factors identifiable from electronic administrative sources alone, thus facilitating design of decision support tools that provide immediate feedback to the practitioner. This approach does not require complex clinical assessments, which in DLBCL may reflect symptom burden rather than baseline functional reserve.

The identified predictors of early death fall into 3 domains related to the lymphoma (B symptoms), comorbidities (kidney disease, prior hospitalization, prior endoscopy), and baseline functional capacity (PS, use of mobility aids). Age of 75 years or older affects all those domains, because it correlates with the DLBCL cell of origin and is a component of the enhanced International Prognostic Index (IPI) ${ }^{29,39}$ The association with endoscopy was somewhat surprising, because endoscopy may not be causally related to the DLBCL. Because this procedure is commonly performed in the workup for anemia, gastrointestinal bleeding, or weight loss, it may correlate with patient's poor nutritional status, abdominal pathology, or low hemoglobin. These factors are otherwise not discernible in claims data, but may affect tolerance of chemotherapy. For example, low mini nutrition- al assessment scores correlate with the risk of early death among older patients receiving chemotherapy for cancer. ${ }^{37}$ In our population of Medicare beneficiaries considered eligible for multi-agent immunochemotherapy, more than half had a reassuringly low risk $(<1 \%)$ of immediate fatal toxicity with curative R-CHOP-like regimens. Notably, this included $40 \%$ of patients aged 80 years or older. Our results suggest that withholding anthracycline may not be associated with additional decrease in risk, as long as adequate cardiac function has been confirmed.

This exploratory study cannot provide direct support for any specific intervention aimed at decreasing the risk of death among patients with DLBCL, but provides direction for further research. Higherrisk groups may be a good target for interventions such as the "prephase treatment" consisting of 7 days of steroid alone before R-CHOP, which decreases lymphoma-related symptoms, improves functional status, and reduces deaths during the first treatment cycle. ${ }^{1,40}$ Alternatively, for the high-risk group, less toxic regimens such as $\mathrm{R}$-mini- $\mathrm{CHOP},{ }^{41}$ rituximab/ bendamustine, ${ }^{42}$ and others, may be preferred to avoid early mortality. ${ }^{31}$ Relevant suggestions could be studied in the context of decision support tools based on automated analysis of the electronic medical record at the time of chemotherapy ordering. We note here that our model is applicable to patients clinically selected for R-CHOP-like chemotherapy, 

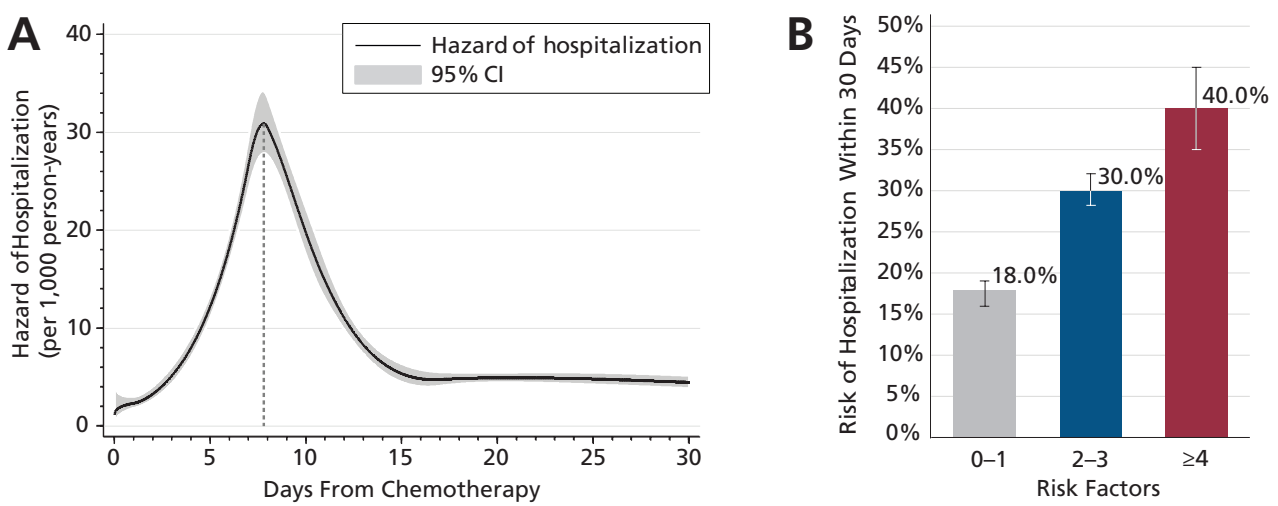

Figure 3. Analysis of the risk of hospitalization in older patients with diffuse large B-cell lymphoma treated with rituximab-based immunochemotherapy. (A) Hazard of hospitalization as a function of time, indicating a peak around day 8 after the first treatment. (B) Risk of hospitalization within 30 days of first treatment, stratified by number of risk factors; bars indicate $95 \% \mathrm{Cls}$.

and may not be optimal for those requiring less intense regimens.

Our results also have some immediate implications for practice. Routine clinical evaluation on days 5 through 7 after the initial chemotherapy can identify impending complications before the peak risk of hospitalization; more than $30 \%$ patients did not receive prophylactic G-CSF. Recently, ASCO tempered the recommendation for G-CSF use in older patients with DLBCL, suggesting only "considering" it and acknowledging uncertain benefit over prophylactic antibiotics. ${ }^{24}$ Our analysis suggests a strong association between omission of G-CSF and early fatalities in the high-risk group, although it is retrospective and not causal. The $66 \%$ rate of G-CSF use was not sensitive to the guarantee-time window or to excluding hospitalized patients, so additional research is warranted on the effect of prophylactic G-CSF in elderly patients undergoing R-CHOP.

An important limitation of our study is that SEER-Medicare does not identify doses of chemotherapy, and therefore we could not determine whether patients had dose reductions. We also could not analyze other laboratory or clinical toxicities. Lack of data on cardiac function or reasons for substituting anthracycline limits the interpretation of the finding that anthracycline use was not associated with excess mortality. Additionally, the IPI or common laboratory parameters might significantly improve the risk stratification. Conversely, some claims-based indicators may be highly specific to Medicare coding, but Medicare beneficiaries constitute a vast majority of older patients with DLBCL in the United States. Our exclusion of managed care plan enrollees and patients treated with lowerintensity regimens may, however, limit the applicable population, and underscores the need for external validation of our results.

\section{Conclusions}

We demonstrated that administrative and cancer registry data, routinely integrated within modern electronic medical records, might be leveraged to stratify the risk of early death among older patients with DLBCL. Although multidimensional geriatric assessments represent a valuable holistic approach to such a stratification, we believe that the use of readily available resources may facilitate adoption of decision support tools into wider practice. Our results provide evidence-based support for safety of standard anthracycline-based immunochemotherapy when the calculated risk is very low, as is the case for more than half of older patients. Because attenuated regimens may impact disease control in the long run, this simple risk stratification might serve as a platform for future interventional studies evaluating deintensified therapy for patients at the highest risk for early toxicity.

\section{Acknowledgments}

This study used the linked SEER-Medicare database. The interpretation and reporting of these data are the sole responsibility of the authors. The authors acknowledge the efforts of the NCI; the Office of Research, Development and Information, CMS; Information Management Services, Inc.; and the 
Early Death in Older Patients With DLBCL

SEER Program tumor registries in the creation of the SEER-Medicare database.

\section{References}

1. Pfreundschuh M. How I treat elderly patients with diffuse large B-cell lymphoma. Blood 2010;116:5103-5110.

2. Fields PA, Linch DC. Treatment of the elderly patient with diffuse large B cell lymphoma. Br J Haematol 2012;157:159-170.

3. Boslooper K, Kibbelaar R, Storm H, et al. Treatment with rituximab, cyclophosphamide, doxorubicin, vincristine and prednisolone is beneficial but toxic in very elderly patients with diffuse large B-cell lymphoma: a population-based cohort study on treatment, toxicity and outcome. Leuk Lymphoma 2014;55:526-532.

4. Delarue R, Tilly $\mathrm{H}$, Mounier $\mathrm{N}$, et al. Dose-dense rituximab-CHOP compared with standard rituximab-CHOP in elderly patients with diffuse large B-cell lymphoma (the LNH03-6B study): a randomised phase 3 trial. Lancet Oncol 2013;14:525-533.

5. Coiffier B, Lepage E, Briere J, et al. CHOP chemotherapy plus rituximab compared with CHOP alone in elderly patients with diffuse large-B-cell lymphoma. N Engl J Med 2002;346:235-242.

6. Habermann TM, Weller EA, Morrison VA, et al. Rituximab-CHOP versus CHOP alone or with maintenance rituximab in older patients with diffuse large B-cell lymphoma. J Clin Oncol 2006;24:3121-3127.

7. Pfreundschuh M, Schubert J, Ziepert M, et al. Six versus eight cycles of bi-weekly CHOP-14 with or without rituximab in elderly patients with aggressive CD20+ B-cell lymphomas: a randomised controlled trial (RICOVER-60). Lancet Oncol 2008;9:105-116.

8. Hamlin PA, Satram-Hoang S, Reyes C, et al. Treatment patterns and comparative effectiveness in elderly diffuse large B-cell lymphoma patients: a surveillance, epidemiology, and end results-medicare analysis. Oncologist 2014;19:1249-1257.

9. Hutchins LF, Unger JM, Crowley JJ, et al. Underrepresentation of patients 65 years of age or older in cancer-treatment trials. N Engl J Med 1999;341:2061-2067.

10. Kim J, Hurria A. Determining chemotherapy tolerance in older patients with cancer. J Natl Compr Canc Netw 2013;11:1494-1502.

11. Gladman JR, Conroy SP, Ranhoff AH, Gordon AL. New horizons in the implementation and research of comprehensive geriatric assessment: knowing, doing and the 'know-do' gap. Age Ageing 2016;45:194-200.

12. Spina M, Balzarotti M, Uziel L, et al. Modulated chemotherapy according to modified comprehensive geriatric assessment in 100 consecutive elderly patients with diffuse large B-cell lymphoma. Oncologist 2012;17:838-846.

13. Olivieri A, Gini G, Bocci C, et al. Tailored therapy in an unselected population of 91 elderly patients with DLBCL prospectively evaluated using a simplified CGA. Oncologist 2012;17:663-672.

14. Extermann M, Boler I, Reich RR, et al. Predicting the risk of chemotherapy toxicity in older patients: the Chemotherapy Risk Assessment Scale for High-Age Patients (CRASH) score. Cancer 2012;118:3377-3386.

15. Olszewski AJ, Winer ES, Castillo JJ. Improved survival with rituximabbased chemoimmunotherapy in older patients with extranodal diffuse large B-cell lymphoma. Leuk Res 2014;38:866-873.

16. Hershman DL, McBride RB, Eisenberger A, et al. Doxorubicin, cardiac risk factors, and cardiac toxicity in elderly patients with diffuse B-cell nonHodgkin's lymphoma. J Clin Oncol 2008;26:3159-3165.

17. Odejide $O O$, Cronin $A M$, Davidoff $A J$, et al. Limited stage diffuse large B-cell lymphoma: comparative effectiveness of treatment strategies in a large cohort of elderly patients. Leuk Lymphoma 2015;56:716-724.

18. Williams JN, Rai A, Lipscomb J, et al. Disease characteristics, patterns of care, and survival in very elderly patients with diffuse large B-cell lymphoma. Cancer 2015;121:1800-1808.

19. Klabunde CN, Potosky AL, Legler JM, Warren JL. Development of a comorbidity index using physician claims data. J Clin Epidemiol 2000;53:1258-1267.

20. Olszewski AJ, Castillo JJ. Comparative outcomes of oncologic therapy in gastric extranodal marginal zone (MALT) lymphoma: analysis of the SEER-Medicare database. Ann Oncol 2013;24:1352-1359.
21. Davidoff AJ, Gardner LD, Zuckerman IH, et al. Validation of disability status, a claims-based measure of functional status for cancer treatment and outcomes studies. Med Care 2014;52:500-510.

22. Davidoff AJ, Zuckerman IH, Pandya N, et al. A novel approach to improve health status measurement in observational claims-based studies of cancer treatment and outcomes. J Geriatr Oncol 2013;4:157-165.

23. Centers for Medicare \& Medicaid Services. Berenson-Eggers Type of Service (BETOS). Available at: https://www.cms.gov/Medicare/Coding/ HCPCSReleaseCodeSets/BETOS.html. Accessed January 20, 2016.

24. Smith TJ, Bohlke K, Lyman GH, et al. Recommendations for the use of WBC growth factors: American Society of Clinical Oncology clinical practice guideline update. J Clin Oncol 2015;33:3199-3212.

25. White IR, Royston P, Wood AM. Multiple imputation using chained equations: issues and guidance for practice. Stat Med 2011;30:377-399.

26. Royston P, Lambert PC. Flexible Parametric Survival Analysis Using Stata: Beyond the Cox Model. College Station, TX: Stata Press; 2011.

27. Giobbie-Hurder A, Gelber RD, Regan MM. Challenges of guarantee-time bias. J Clin Oncol 2013;31:2963-2969.

28. Ziepert M, Hasenclever D, Kuhnt E, et al. Standard International prognostic index remains a valid predictor of outcome for patients with aggressive CD20+ B-cell lymphoma in the rituximab era. J Clin Oncol 2010;28:2373-2380.

29. Zhou Z, Sehn LH, Rademaker AW, et al. An enhanced International Prognostic Index (NCCN-IPI) for patients with diffuse large B-cell lymphoma treated in the rituximab era. Blood 2014;123:837-842.

30. Feugier P, Van Hoof A, Sebban C, et al. Long-term results of the R-CHOP study in the treatment of elderly patients with diffuse large B-cell lymphoma: a study by the Groupe d'Etude des Lymphomes de l'Adulte. J Clin Oncol 2005;23:4117-4126.

31. Morrison VA, Hamlin P, Soubeyran P, et al. Approach to therapy of diffuse large B-cell lymphoma in the elderly: the International Society of Geriatric Oncology (SIOG) expert position commentary. Ann Oncol 2015;26:1058-1068.

32. Mora O, Zucca E. Management of elderly patients with hematological neoplasms. Ann Oncol 2007;18(Suppl 1):i49-53.

33. Terschuren C, Gierer S, Brillant C, et al. Are patients with Hodgkin lymphoma and high-grade non-Hodgkin lymphoma in clinical therapy optimization protocols representative of these groups of patients in Germany? Ann Oncol 2010;21:2045-2051.

34. van der Poel MW, Mulder WJ, Ossenkoppele GJ, et al. Factors that influence treatment decision-making in elderly DLBCL patients: a case vignette study. Ann Hematol 2015;94:1373-1379.

35. Thieblemont $\mathrm{C}$, Grossoeuvre A, Houot R, et al. Non-Hodgkin's lymphoma in very elderly patients over 80 years. A descriptive analysis of clinical presentation and outcome. Ann Oncol 2008;19:774-779.

36. Extermann M, Hurria A. Comprehensive geriatric assessment for older patients with cancer. J Clin Oncol 2007;25:1824-1831.

37. Soubeyran P, Fonck M, Blanc-Bisson C, et al. Predictors of early death risk in older patients treated with first-line chemotherapy for cancer. J Clin Oncol 2012;30:1829-1834.

38. Hurria A, Togawa K, Mohile SG, et al. Predicting chemotherapy toxicity in older adults with cancer: a prospective multicenter study. J Clin Oncol 2011;29:3457-3465.

39. Mareschal S, Lanic H, Ruminy P, et al. The proportion of activated B-cell like subtype among de novo diffuse large B-cell lymphoma increases with age. Haematologica 2011;96:1888-1890.

40. Pfreundschuh M, Trumper L, Kloess M, et al. Two-weekly or 3-weekly $\mathrm{CHOP}$ chemotherapy with or without etoposide for the treatment of elderly patients with aggressive lymphomas: results of the NHL-B2 trial of the DSHNHL. Blood 2004;104:634-641.

41. Peyrade F, Jardin F, Thieblemont C, et al. Attenuated immunochemotherapy regimen $(\mathrm{R}$-miniCHOP) in elderly patients older than 80 years with diffuse large B-cell lymphoma: a multicentre, single-arm, phase 2 trial. Lancet Oncol 2011;12:460-468.

42. Walter E, Schmitt T, Dietrich S, et al. Rituximab and bendamustine in patients with CD20+ diffuse large B-cell lymphoma not eligible for cyclophosphamide, doxorubicin, vincristine and prednisone-like chemotherapy. Leuk Lymphoma 2012;53:2290-2292. 